Voix et Images

voixetimages

\title{
Le lisible et ses ombres
}

\section{André Brochu}

Volume 11, numéro 3 (33), printemps 1986

Yolande Villemaire

URI : https://id.erudit.org/iderudit/200591ar

DOI : https://doi.org/10.7202/200591ar

Aller au sommaire du numéro

\section{Éditeur(s)}

Université du Québec à Montréal

\section{ISSN}

0318-9201 (imprimé)

1705-933X (numérique)

Découvrir la revue

\section{Citer cet article}

Brochu, A. (1986). Le lisible et ses ombres. Voix et Images, 11(3), 555-563.

https://doi.org/10.7202/200591ar d'utilisation que vous pouvez consulter en ligne.

https://apropos.erudit.org/fr/usagers/politique-dutilisation/ 


\title{
Poésie
}

\section{Le lisible et ses ombres}

\author{
par André Brochu, Université de Montréal
}

Une poésie qui ne vise pas à la qualité de chef-d'œuvre n'est pas pour autant une poésie sans mérites; son absence de prétention ne l'empêche pas de véhiculer d'intenses émotions: ironie, tendresse, indignation, amertume, regret - tous rayons du soleil amour. Dans ses Poèmes sauvés du monde, Yves Boisvert ${ }^{1}$, qui ne se pose nullement en rédempteur de l'humanité, tente plutôt de créer de petits havres de langage où mettre à l'abri ce qui peut être sauvé de ses espoirs et de ses souvenirs, des nôtres aussi. Il y a le départ del'être aimé, avec promesses de retrouvailles quand tu auras quarante-cinq ans et tu feras du rhumatisme / nous rivaliserons de fistules et de chancres. Il y a le marasme présent:
Ce soir
le ciel clignote de petits désespoirs ennuyants
le ciel tourne au rougeoiement du bégonia hurleur et tout semble rester calme.

Une violence sans apprêt, qui ne dédaigne pas la saillie vulgaire, imprègne le rapport au quotidien. Le langage est dru, direct, rarement embelli d'images émerveillantes mais jamais terne pour autant. On y trouve quelques incorrections (non voulues), par exemple cette tournure vicieuse dont raffole aussi François Charron: pour ne pas que.... Mais le discours poétique est généralement dense, prenant, chargé d'intensité dramatique (le poète s'adresse souvent à la femme aimée, qui est absente) plus que de complexes résonances signifiantes. On y trouve des strophes qui transfigurent le poème, qui le tirent de ses allures canailles pour, tout à coup, en agrandir les perspectives:

L'air est moins rare à ton niveau

de ton corps on peut voir très loin à l'avance

au delà des lignes de feu et des avalanches

au delà des rives interdites des saisons

l'air est moins rare et plus stricte la coupure

et plus acérée la limite des paysages.

Le dernier vers montre comment la trouvaille peut jaillir des mots - ou ne serait-ce plutôt des idées? - les plus simples; mais il faut aussi louer la paronomase à l'avance / avalanches et la belle cascade de dentales, aggravée par les doubles génitifs, des vers 3 et 4 .

Une poésie qui a quelque chose à dire, et qui le dit mieux qu'avec «bonheur»: avec liberté. 
Les petits blocs de prose qui composent le dernier recueil de Hugues Corriveau, Forcément dans la tête ${ }^{2}$, n'accrochent pas tout de suite le lecteur. Ce dernier doit d'abord se rendre attentif au retour de certains mots, plaisir, désir, mort. Et tous ceux qui gravitent autour: avaler, mou, peau, froid; mais aussi enfant, amour, frère. Ces mots, qui ne sont pas tout à fait des choses (ou des êtres), jouent, se rencontrent, composent entre eux ou se fuient. Et peu à peu, très lentement, de ce langage apparemment insensible ou insensé, appliqué, trop beau pour être vrai, émergent quelques images d'horreur vive, à même les mots patiemment combinés et jusque-là dépourvus de référence. Voilà que se dévoile le réel, un réel digne de Apocalypse now, celui-même qui risque de signaler notre sièclé à la réprobation de l'humanité future (s'il en est):

On brûle, vivant, en pleine rue, on lapide aussi le long des voies ferrées parce qu'une femme est morte. Ailleurs, on fait éclater des crânes d'enfant pour que la paix du jour ne fasse plus de bruit avec leurs cris, leurs jeux. On noie des femmes dans le sang de leur ventre, on coupe des têtes qu'on transporte comme des trophées dans des paniers à légumes. (...)

Sans doute la représentation est-elle, d'une certaine façon, excessive et gorgée de fantasmes. La dénonciation de la folie et de l'horreur collectives a de sourdes motivations individuelles: le jeu du je n'est pas complètement perdu de vue. Mais une sortie vers le réel est effectuée. Si ce livre a quelque sens, il va là où se parle la peur de mourir. Pas seulement celle de l'auteur mais de tous. Et peu de poèmes dits engagés sont aussi convaincants, car Corriveau ne cesse jamais d'être écrivain, dresseur de mots. Pas redresseur de torts ou prophète en images. Un homme pour qui tout se passe "forcément dans la tête». Un homme de langage — habité par le monde.

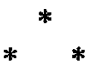

Bernard Pozier, qui a peut-être déjà eu de l'inspiration mais qui changeait de style comme de chemise, troquant le blouson du rocker pour le sarrau des sciences à la mode, nous offre à la fois un condensé et une caricature de son savoir-faire poétique dans ses Bacilles de tendresse ${ }^{3}$. Deux titres de section, "Textes au laser» et "Textes au latex», rappellent d'ailleurs le cuir (imité) et la sophistication technologiste des recueils précédents. Mais ici tout est mêlé, l'hobo et le robot, le logiciel et la tarentelle. Après d'autres, Pozier assume à son tour le pari du lisible / le risque du lyrisme. Il choisit donc de délaisser les lentes et tumultueuses explorations pour revenir parler dans l'écriture / presque simplement. Le presque est bien honnête; car Pozier a décidé de poétiser son langage à grands renforts de paronomases, ce qui l'éloigne de la simplicité pure! Cette technique est fort répandue dèpuis qu'on s'entend à faire la preuve, noir sur blanc, qu'on s'y connaît en plaisir-du-texte. Voulez- 
vous de l'homophonie anaphorisante (disons: de l'anaphonie)? En voilà: cadran cadraste calendrier calcul calepin. Tout commence par ca: ca.... ca... Un dictionnaire ne ferait pas mieux. Voulez-vous du calembour (encore un mot en ca!)? Tcheckez bien: néo / roman / tics. À mettre en rapport avec cet art poétique:

et sur l'écran cathodique de la fin du siècle

l'inscription voyante d'un nouveau romantisme

l'écriture dévorante de l'absolue tendresse

Les bacilles de la tendresse sont sans doute proches parents des enzymes gloutons puisqu'ils soumettent le langage à une constante analyse. Les mots se présentent couplés à leur sosie, minés, minables:

ses cheveux ses chevilles

ses cuirs ses cuisses

(...)

s'eclatent

s'écartent

s'écarlatent

Ce n'est pas là du travail sur le signifiant mais un jeu - insignifiant. Un jeu qui ne suffit certes pas à faire exister littérairement les lieux communs que le poète se donne pour sujets, lui qui - je cite une dernière ineptie - veut choisir le vers facile / contre le solitaire.

Dommage: la tendresse n'a pas encore trouvé son champion fin-de-siècle.

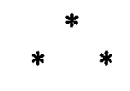

À l'heure où plusieurs poètes dans la trentaine avancée confessent leur désillusion et leur amertume devant la rareté de leurs lecteurs ou le peu de cas que fait d'eux la relève, d'autres poursuivent sereinement leur œuvre et publient leurs meilleurs titres. C'est le cas de Claude Beausoleil, qui fait paraître un livre remarquable, S'inscrit sous le ciel gris en graphiques de feut. Ce vaste poème de plus de trois mille vers est dédié à Clément Marchand, qui a l'un des premiers chanté la ville dans les poèmes des Soirs rouges (1947). Un poème de Marchand, "les Prolétaires», contenait l'alexandrin suivant: $S$ 'inscrit sous le ciel gris en graphiques de fer. Beausoleil le reprend en le modifiant légèrement et en fait non seulement le titre mais l'inspiration même de son texte, dont la ville et l'écriture sont les thèmes omniprésents. Sur le plan formel aussi, Beausoleil renoue à sa façon avec la métrique traditionnelle, fondée sur la régularité stemmatique, tout en proposant des règles nouvelles et un rythme personnel qui conjurent le risque de platitude. Le texte comporte une seule coulée de vers assez brefs, semblable à ce qu'on trouvait déjà dans

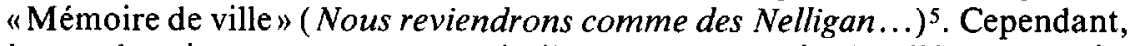
à tous les cinquante vers un quintil, dont une partie des éléments varie, renoue le poème à lui-même, marquant à la fois la continuité et l'extraordi- 
naire diversité d'une inspiration vaste et une comme la ville elle-même, comme l'écriture toujours recommencée. Le premier quintil se lit ainsi:

le poème est un objet sensible

et c'est l'image du monde

la ville le traverse

et c'est le rythme qui saccade

comme dans le souffle des découvertes

et voici le dernier, qui intègre les mots de Clément Marchand (je souligne):

le poème est une origine

et c'est l'image du ciel

la ville l'inscrit

et c'est le rythme des graphiques

comme dans le souffle du fer

Entre les deux, cinquante-trois variations du quintil sont autant de machines de rêve ouvertes à l'infini du sens, du réel, de l'existence. Jamais Beausoleil n'a été plus inventif, plus constamment intéressant, nous touchant par son langage direct, chaleureux, vif. Langage passablement classique du reste si l'on considère la métrique: très peu, sinon pas de rejets ou de contrerejets, chaque vers est une proposition ou un noyau complet de proposition, ce qui ajoute à la lisibilité - une lisibilité qui n'est jamais une facilité, même si les vers de Beausoleil n'ont rien de «solitaires»!

Malgré son ampleur, il faut le noter, le poème ne relève pas du genre épique car il ne comporte aucune mystification héroïque. J'y verrais plutôt une "méditation», où les rapports entre ville et écriture sont explorés par la conscience rêveuse et la conscience réflexive sans qu'on tombe jamais dans le didactisme ou l'onirisme gratuit. D'émouvants souvenirs soutiennent l'évocation du montréal d'enfance de collège / montréal d'amour et d'écriture, mais la veine autobiographique s'efface à point nommé devant une inspiration plus universelle, et se découvre alors Montréal cosmique et monochrome / sous ces effets sifflants / qui donnent à tout des airs / mimant la fulgurance. Jamais on n'avait ainsi chanté Montréal, avec des accents aussi fervents que d'autres poètes avaient trouvés pour dire le pays, mais une liberté, une modernité, une universalité d'inspiration peu souvent égalées.

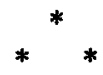

Il faut parfois du temps avant que le cuivre ne s'éveille clairon. Tout est en place, les mots, les thèmes, la folie nécessaire; il manque simplement le petit rien qui change la page en poème, les images en actes. Une fois pour toutes.

C'est ce qui vient d'arriver à Paul Chanel Malenfant. Peut-être pas encore dans les Noms du père ${ }^{6}$, qui compte pourtant de belles et terribles évocations de ce qui se glisse de silences, de malentendus, de tensions entre 
père et fils et de leurs reconductions dans l'existence quotidienne, jalonnée d'amours empêchées. Mais, à coup sûr, dans le petit recueil intitulé En tout état de corps ${ }^{7}$. Pour la première fois le voile du temple est déchiré, la vérité est montrée à vif. Finies les habiletés, les pudeurs, les gentillesses: un homme, qui est un enfant, face à la mort (la mère, le père, leur insoutenable relation), parle désormais le langage de son destin. Il trouve, très simplement, des mots - des rythmes - inouis:

Il tremble en des effrois de laitue et de dimanche.

...

(Et ma mère tue mes chats, fane les noix: purée de cris.)

...

(...) et j'ai des larmes jusqu'aux genoux quand le père lit à voix basse (...)

..

La même femme et même mort. Émeute ou mime. Je palpe et parle: au nom du père et du fils et père, pour écrire, me prend par la main.

Faut-il faire remarquer, dans la dernière citation, le jeu des $\mathbf{m}$ (massés dans les deux premières phrases consacrées à la mère) et des $\mathbf{p}$ (rapportés au père)?

La lecture de En tout état de corps, qui me découvre une voix enfin personnelle, délivrée des réminiscences, me permet du même coup de situer l'idiome-Malenfant par rapport à d'autres, plus connus du lecteur. Deux noms me viennent à l'esprit, même si leur conjugaison ne saurait expliquer une voix aussi singulière: Rina Lasnier (que Malenfant connaît bien: il lui a consacré son mémoire de maîtrise), pour l'extrême dignité du langage, peutêtre quelque onction; et Anne Hébert, pour l'acuité du sentiment et de l'image. Et l'une et l'autre pour la passion d'écrire, à contre-ciel et à contre-enfer.

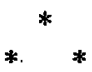

Dans cette chronique où l'espace m'est mesuré pour faire état des bons recueils qui paraissent, il me faut tout de même signaler Nobody de Carole Massé 8 . Ce roman trouverait difficilement sa place ailleurs car il se rapproche trop du genre poétique, si tant est que la poésie soit un genre et que le roman ne puisse prendre la forme qu'il lui plaît. D'ailleurs je ne veux nullement minimiser les dons de narratrice de Carole Massé. Mais cette quête des origines, du sens de l'existence dont son récit est l'invention passionnée, soumet la représentation romanesque, si articulée soit-elle, à une logique du désir qui débouche tout droit sur le poème. Elle l'écrit elle-même: ... n'avais-je pas bifurqué vers la poésie, affranchie donc d'un sens, d'un salut, d'un rassasiement, livrée sans espoir à l'enchevêtrement du hasard et du désir?

Plusieurs passages réaffirment le lien entre désir, poésie, infini, enchevêtrement du réel et du rêve. La remontée vers les origines est déclenchée par la 
mort de la mère. Il y a d'abord le débondement des sentiments amoureux de la narratrice à l'égard de celle qui lui a donné la vie et qui devient, par le pouvoir de l'imaginaire, sa contemporaine. Puis l'enquête de la narratrice s'élargit jusqu'à englober le père, qui est anglophone malgré son nom de Hébert. Le "Couple» des «géniteurs" est rêvé sur le mode de l'amour fou et de la beauté incarnée. L'anglais tient une place de plus en plus grande dans le discours narratif et triomphe dans un appendice au récit, Poems for the Dead Children, signé Carole Hébert (Massé étant le patronyme maternel).

Cette longue fantaisie autobiographique où les événements intérieurs, anamorphosés par le rêve, ont une place prépondérante, présente de grandes qualités d'écriture. Ce qui m'apparait remarquable, en particulier, c'est la fusion de deux problématiques jusqu'ici ennemies, celle de la quête des origines avec toutes ses résonances symboliques et individuelles, et celle de la modernité impliquant l'éclatement du temps, de la représentation et de l'identité (le titre, Nobody, indique la désappartenance radicale au monde, explique le prière d'insérer). On ne sent aucun hiatus entre ces champs signifiants, non plus qu'entre la forme romanesque et le contenu "poétique" qui trouve du reste son expression spécifique dans les poèmes anglais de la fin. D'ailleurs l'anglais "paternel» et le français «maternel» font aussi bon ménage que les "géniteurs" eux-mêmes; et cette diglossie d'auteur ne détonne nullement dans le présent contexte culturel. L'anglais, dans les romans et les poèmes qui s'écrivent aujourd'hui au Québec, joue sans doute un rôle analogue à celui du joual au cours des années 60 . Il exprime notre vérité la plus profonde, notre radicale désappartenance, épurée cette fois de tout vestige maternel.

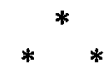

Le deuxième recueil de Michel Savard, Cahiers d'anatomie ${ }^{9}$, confirme la maîtrise du langage poétique qui avait valu à l'auteur de Forages ${ }^{10}$ deux prix prestigieux. Ce ne sont pas tant les images, ni l'exploitation ingénieuse des sonorités qui font la qualité des poèmes de Michel Savard mais un mélange de désinvolture et de calcul, un tact infini. La tension lisible-illisible, inhérente au texte, est souvent plus poussée que chez certains formalistes de naguère, maintenant convertis à la romance. On est parfois près de l'hermétisme laconique d'un Renaud Longchamps; d'autres fois, la trivialité reprend ses droits et des vers comme ceux-ci:

\author{
Un jour c'était \\ le second match de la série contre les Phillies \\ de Philadelphie la balle file ma douceur \\ à plus de cent milles l'heure
}

ne seraient pas déplacés dans les recueils récents du regretté Michel Beaulieu. Mais les dangers sont soigneusement conjurés, le poème retombe sur ses pattes comme un chat, faisant valoir ses rythmes-émotions, ses évocations de 
vie du moi et de vie du couple et d'amours immensément quotidiennes, jamais prévisibles, semées de miracles et de déceptions, d'obscénités et de tendresses.

Aucun des cent vingt-six poèmes (sans titre) n'a plus d'une page, mais l'homogénéité de l'ensemble est assurée par le retour de deux incipits: un jour c'était (trente et une fois) et que dire que dire (dix-huit fois). Le premier annonce un événement, le second un commentaire - l'un, des mots; l'autre, des choses; et ces deux ordres de signification se partagent à parts égales un discours que menacent peut-être la préciosité, l'inutile ou le procédé mais qui, le plus souvent, est charme et séduction.

Clos mon univers! s'exclame Anne-Marie Alonzo au seuil de Bleus de mine ${ }^{11}$. Et pourtant on y entre aisément, accroché tout de suite par une voix, une angoisse, un style. On dirait un monologue pour la scène, même si le texte est totalement écrit, avec ses effets de syntaxe qu'on pourrait qualifier de savants. Rien de déclamatoire, de platement transitif ou référentiel. Cependant c'est une voix qui se construit, se tend, se querelle, qui dit le chant de soi et de l'autre, de l'amour et de la mort, de l'enfance incertaine et forclose (l'enfance s'appelle Alexandrie). Une voix où se bousculent les mots comme une troupe de travestis, au genre et au nombre erratiques:

De vive alexandrie se perd la grande pays s'épuise amie pays se meurt et meurent ensemble souvenance souvenue.

Langage très performatif: chaque vers est un acte, un geste, une création arrachée aux conventions du langage comme du bien-vivre. Condamnée par l'infirmité à une dépendance cruelle, Anne-Marie Alonzo réinvente avec courage son rapport au monde, aménage une existence viable, trouve en l'amour la force de supporter le terrible exil auquel la contraignent non seulement la perte du pays d'enfance mais aussi celle du mouvement:
Alors et yeux fermés j'invente rappelle
est-ce vrai petite fois ou toujours
recrée y avait enfance en pays chaud
bougeaient aussi les jambes et corps
arrondi formé et déjà né d'absence.

La nostalgie est incontournable car le passé est la seule référence à partir de quoi peut s'écrire l'aujourd'hui. Mais le poète ne s'y enferme pas, au contraire. À la clôture de son univers, dont le constat ouvrait le livre, répond la répudiation finale du mirage alexandrin:

Ailleurs bien ailleurs cette fuite hors d'égypte. 


\section{VOIX \& IMAGES/33, printemps 1986}

Ambre gris, deuxième recueil de Michel Lemaire ${ }^{12}$, fait entendre une voix inhabituelle dans notre poésie. Sans doute est-elle l'écho des origines françaises de l'auteur; et de ses préoccupations pour des mouvements sociolittéraires qui nous sont peu familiers, comme le dandysme (auquel il a consacré un savant ouvrage). Quoi qu'il en soit, Ambre gris - le gris est la couleur préférée du dandy - est un véritable régal littéraire, une poésie très articulée, fort éloignée du vague à l'âme même si le poète, moderne des Esseintes, n'a rien d'un joyeux drille. Mais son désespoir intime est l'origine d'un savoir précis, dont l'expression ne court pas le risque de la répétition:

Les mots ne sont jamais les mêmes

Ni ceci ni cela, ni pour toi ni pour moi,

Ni pour la difficulté d'être.

Malgré tout.

Le néant de l'existence est peuplé d'une foule de petits riens, dont l'énumération constitue l'activité poétique par excellence. Tout est énumération, comme le suggèrent déjà les titres des sections: "Cubismes", "Filles», "Chronologies». Le recueil est une collection d'aperçus, de souvenirs amoureux, d'instants revécus, et chacun est constitué d'une addition de touches très concrètes. Par exemple:

Les bruits du monde, les gratte-ciel

Dans le couchant, la lune où l'on ira,

Le téléjournal, la soirée du hockey,

Les brûlures, les merveilles, l'oiseau crevé,

Qui est à l'appareil? Ennuyé, cravaté,

Qui cherche son chemin, si près de renoncer?

Traîtrises coulantes, esthétiques, mégots,

Gyroscope affolé.

L'expression du sentiment résulte du heurt des multiples aspects du vécu, dont on voit bien qu'aucun n'accapare le désir: tout est convoyé vers la mort, sans que celle-ci soit nommée. Elle est simplement la vitesse des choses, l'impossibilité qu'elles soient sujets de développements. Tout défile sans retour, chaque vers est une exécution.

L'ambre gris est une concrétion intestinale du cachalot, qui sert à composer des parfums précieux. Les poèmes de Lemaire font ainsi l'effet d'être les concrétions d'une existence à ses thèmes; mais leur accumulation serait le phore magnifiant — d'une parfaite discrétion.

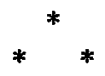

J'ai peu de choses à dire de Quartz et mica, de Yolande Villemaire ${ }^{13}$. J'aime assez le parti pris de simplicité de l'écriture, le rythme ample et serein des vers, mais leur prosaïsme, peu importe qu'il soit voulu ou non, me déçoit. On sent que l'auteur entend être fidèle à son expérience, qu'elle refuse de 
l'orner; mais cette expérience, précisément, n'a rien à voir avec la poésie. Elle relève d'une spiritualité ésotérique, habile à exploiter ce que j'appellerai le sacré à sensation: Atlantide, vies antérieures, etc. L'humour, hélas, n'illumine plus les textes de Villemaire. Son évocation de New York est bien pâle à côté des fresques lyriques qu'un Beausoleil a consacrées à la ville (Montréal). Quant à l'ésotérisme, quelques auteurs ont prouvé qu'il pouvait, malgré tout, servir une intention poétique.

Souhaitons que cette éclipse du sens littéraire ne dure pas.

1. Yves Boisvert. Poèmes sauvés du monde, Trois-Rivières, Écrits des Forges, 1985, $72 \mathrm{p}$.

2. Hugues Corriveau, Forcément dans la tête, Montréal, les Herbes rouges, 1985, $90 \mathrm{p}$.

3. Bernard Pozier, Bacilles de tendresse, Trois-Rivières, Écrits des Forges, 1985, $110 \mathrm{p}$.

4. Claude Beausoleil, $S$ 'inscrit sous le ciel gris en graphiques de feu, Trois-Rivières, Écrits des Forges, 1985, $114 \mathrm{p}$.

5. Claude Beausoleil, Une certaine fin de siècle, Saint-Lambert, le Noroît, 1983, pp. 45-57.

6. Paul Chanel Malenfant, les Noms du père suivi de Lieux dits: italique, Saint-Lambert, le Noroît. 1985, 92 p.

7. Paul Chanel Malenfant, En tout état de corps, Trois-Rivières, Écrits des Forges, 1985. $76 \mathrm{p}$.

8. Carole Massé, Nobody, roman, Móntréal, les Herbes rouges, 1985, $150 \mathrm{p}$.

9. Michel Savard, Cahiers d'anatomie (complicités), Saint-Lambert, le Noroît, 1985, $158 \mathrm{p}$.

10. Michel Savard, Forages, Saint-Lambert, le Noroît, 1982, 86 p. Prix du Gouverneur général 1982 et Troisième prix des Jeunes Écrivains du Journal de Montréal.

11. Anne-Marie Alonzo, Bleus de mine. Saint-Lambert, le Noroît, 1985, 68 p.

12. Michel Lemaire, Ambre gris, Saint-Lambert, le Noroit, 1985, 64 p.

13. Yolande Villemaire, Quartz et mica, Trois-Rivières et Pantin (France), Écrits des Forges / le Castor astral, 1985, 54 p. 